\title{
Severe pressure ulcers requiring surgery impair the functional outcome after acute spinal cord injury
}

\author{
Martin Donhauser ${ }^{1} \cdot$ Lukas Grassner $^{1,2,3} \cdot$ Barbara Klein $^{3} \cdot$ Maika Voth $^{4} \cdot$ Orpheus Mach $^{1} \cdot$ Matthias Vogel $^{1} \cdot$ \\ Doris Maier ${ }^{1} \cdot$ Dorien Schneidmueller ${ }^{4,5}$
}

Received: 6 December 2018 / Revised: 26 June 2019 / Accepted: 26 June 2019 / Published online: 16 July 2019

(c) The Author(s), under exclusive licence to International Spinal Cord Society 2019

\begin{abstract}
Study design Retrospective matched cohort study.

Objectives Assessing the influence of surgically managed grade 3 and 4 pressure ulcers (PU) in the acute phase after spinal cord injury (SCI) on the neurological and functional outcome after 1 year.

Setting Specialized SCI-unit within a level 1 trauma center in Murnau, Germany.

Methods We performed a retrospective matched cohort study. For every patient with acute SCI and a PU requiring surgery, we identified matched controls within our database in a 1:3 ratio. Matching criteria were: AIS-grade (American Spinal Injury Association Impairment Scale), neurological level and age. The scores of the SCIM-III (Spinal Cord Independence Measure) and the ISNCSCI (International Standards for Neurological Classification of Spinal Cord Injury) as well as the total length of stay (LOS) at the hospital were used as outcome parameters. We applied a stratified analysis using a conditional logistic regression to test for group differences in each outcome parameter of the study.

Results In a 6-year period (2010-2015) 28 patients required flap surgery due to 3-4 ${ }^{\circ} \mathrm{PU}$ in the acute phase after SCI. Of these patients, 15 had complete data sets according to the EMSCI (European Multicenter Study about Spinal Cord Injury) protocol. Patients with severe PUs during the acute SCI phase had a significantly impaired functional outcome. After 1 year the improvement of the SCIM score was significantly lower in the PU group compared to the control group (17.4 versus $30.5 ; p<0.006)$. However, the change in AIS grade after 1 year was not significantly affected. The LOS was prolonged by a mean of 48 days in the PU group $(p<0.006)$.

Conclusions Severe PUs requiring surgery in the acute phase after SCI impair the functional outcome and increase LOS. Preventive measures should be applied to all acute SCI patients. Patients should be transferred to specialized SCI-centers as soon as possible.
\end{abstract}

Martin Donhauser

martin.donhauser@bgu-murnau.de

1 Center for Spinal Cord Injury, Trauma Center Murnau, Murnau am Staffelsee, Germany

2 Department of Neurosurgery, Medical University Innsbruck, Innsbruck, Austria

3 Institute of Molecular Regenerative Medicine, Spinal Cord Injury and Tissue Regenerations Center Salzburg, Paracelsus Medical University, Salzburg, Austria

4 Department of Trauma-, Hand- and Reconstructive Surgery, Goethe University Frankfurt, Frankfurt, Germany

5 Departement of Traumatology, Trauma Center Murnau, Murnau am Staffelsee, Germany

\section{Introduction}

Acute spinal cord injury (SCI) has a severe impact on the patient's health and future quality of life. For this reason, specialized therapy including comprehensive acute care and rehabilitation should be started as soon as possible to achieve an optimal outcome. The ultimate goal is to help the patient to return to their former social and professional environment.

Treatment options for SCI remain limited. Current principles of acute care include early surgical decompression, stabilization of the injured spinal column, and blood pressure augmentation during the first week after injury [1-3].

Rehabilitative measures, such as mobilization and locomotion training are also started as early as possible to promote regeneration [4-6]. 
SCI patients are susceptible to complications in particular during the acute phase after injury [7]. Complications within this critical phase are likely detrimental for the functional outcome [8]. An early transfer to a specialized center capable of providing integrated multi-disciplinary care decreases not only the number as well as the severity of complications, but, also overall mortality and the overall total length of stay (LOS) [9].

The occurrence of pressure ulcers (PUs) is a common complication after SCI [8, 10].

Salzberg showed in 1999 that $49 \%$ of patients admitted to a specialized center for acute SCI suffered from PUs [11]. More recent studies have shown that skin complications had a prevalence of $15.6 \%$ after acute SCI [7] or an incidence of $33.5 \%$ after the first 12 months respectively [12].

In the acute phase after SCI, the management of PUs interfers with certain treatment and early rehabilitation paradigms. For every PU, the basic principle of therapy is pressure relief [13], which often results in bed rest. Thus mobilization, locomotion training and other rehabilitative measures are postponed.

Even though many different factors contribute to the outcome of the therapy of acute SCI, there are indications that complications during the early rehabilitation phase, and in particular PUs, have a detrimental effect. Richard-Denis et al. showed in a systematic review that the occurrence of medical complications during acute therapy and rehabilitation is a negative predictive factor for global functional outcome [14]. A study from the Netherlands reported a weak association between a higher functional independence measurement (FIM) score and fewer days of bedrest attributed to pressure ulcers [15].

In this study we investigate the impact of severe PUs occurring during the acute phase after SCI on the functional and neurological outcome after one year. We focused on severe PUs requiring flap surgery, because part of the treatment protocol was a long period of bedrest which postponed rehabilitative measures.

For this, we analyzed differences between patients suffering from severe PUs during the acute SCI phase compared to controls (SCI patients with no Pus) in important neurological and functional outcome parameters in the chronic phase (SCIM score, LOS, and AIS grade after one year). To adjust for possible confounding parameters, a matched study design was chosen (matching was done for baseline AIS grade, neurological level on admission, and age).

\section{Methods}

\section{Setting}

We performed a single-center study at the Trauma Center Murnau (Bavaria, Germany), a cross-regional level 1 trauma center with a specialized SCI department.

\section{Patients}

Within our institutional database we identified 28 patients over a 6-year period (2010-2015) who required 29 flap surgeries due to severe PUs in the early phase after SCI. We matched these patients with patient data in the EMSCI (European Multicenter Study about Spinal Cord Injury) database (a web-based register for lifelong monitoring of SCI-patients) of our hospital. In this prospective database the neurological and functional recovery at predetermined time points after acute SCI is recorded.

The PU group included patients with acute SCI, older than 15 years, with available follow-up who were suffering from severe PUs requiring flap surgery. Severe PUs were defined as grade 3 and 4 according to the international NPUAP/EPUAP Pressure Ulcer Classification System published in the 2014 "Prevention and Treatment of Pressure Ulcers: Quick Reference Guide" provided by NPUAP (North American Pressure Ulcer Advisory Panel), EPUAP (European Pressure Ulcer Advisory Panel) and PPPIA (Pan Pacific Pressure Injury Alliance) [16]. Grade 3 means full thickness skin loss and grade 4 means full thickness tissue loss.

We identified 15 patients which met our inclusion criteria for the PU group. For the control group, we applied the same inclusion criteria, the only difference was that these patients did not suffer from any PU during the acute phase (Table 1).

For our matching process we applied a 1:3 ratio. This means that for every patient with a PU we identified 3 control patients without PU in our database. In order to minimize potential confounders, we controlled for the following parameters: baseline AIS grade, neurological level on admission and age. Special attention was given to the neurological baseline situation, in particular, patients with an cervical injury were matched to exactly the same AIS grade and neurological level as well as comparable TMS (total motor score) values. For thoracic injuries, we accepted discrepancies of 1 or 2 thoracic levels as this only mildly affects sitting stability. Age was grouped in 15-year increments (16-30, 31-45, 46-60, 61-75, 76+).

All patients underwent prospectively a detailed neurological examination according the ISNCSCI protocol

Table 1 Eligibility with inclusion and exclusion criteria

\begin{tabular}{ll}
\hline Inclusion criteria & Exclusion criteria \\
\hline Newly diagnosed SCI & Subacute or chronic phase \\
$\begin{array}{l}\text { PU EPUAP grade 3-4 requiring flap } \\
\text { surgery }\end{array}$ & No PU or grade 1-2 \\
Follow-up data available & Missing follow-up data \\
Age $>15$ & Age $<16$ \\
\hline
\end{tabular}


(International Standards for Neurological Classification of spinal Cord Injury) and an evaluation of the Spinal Cord Independence Measure (SCIM-III) on admission (acute I 16-40d or acute II 70-98d post injury), before discharge (acute III 150-186d post injury) and after 1 year (chronic) [17]. In this study we compared neurological data from the acute I phase with chronic findings.

We chose the SCIM score as the primary outcome parameter. As secondary outcome parameters, we selected the LOS and the AIS grade.

The SCIM was developed specifically for SCI-patients as a global measurement tool assessing activities of daily living and mobility tasks. The SCIM measures parameters which are essential for the patients" daily life, such as respiration, bladder function, bowel management, food intake, personal hygiene, and mobility. The domains self-care, respiration and sphincter management, mobility (room and toilet) and mobility (indoors and outdoors, on even surfaces) were assessed by observer ratings. The SCIM is validated for traumatic as well as non-traumatic SCI. It is a reliable tool for describing long term functional changes [18-20].

Only the SCIM III version was used in this study $[19,20]$, since it is the most recent SCIM version and also part of the EMSCI protocol. All parameters were assessed by specially trained and certified examiners according to the ISNSCI protocol.

Neurological recovery was measured in AIS grade (AIS A-D), neurological level (C1-S5), total motor score (TMS range $0-100$ ) and functional recovery by SCIM (range 0-100).

We analyzed changes in the AIS classification as conversion rate and difference of neurological level [2].

LOS was defined as the time between admission and discharge from our hospital. Being a cross-regional level 1 trauma center with a specialized SCI department our hospital provides treatment for acute SCI continuously from the emergency room to specialized SCI rehabilitation. Acute SCI patients are treated until they reach rehabilitation goals like independent wheelchair mobility and mastering of activities of daily life as well as an established bladder and bowel management.

\section{Procedures}

In our institution all patients with EPUAP grade 3 or 4 in the sacral ischial or trochanteric region are generally treated for PU by flap surgery with the same protocol. Grade 1 and 2 PUs are treated with a less strict immobilization protocol to minimize impact on mobilization and rehabilitation.

All patients were immobilized immediately after diagnosis of a $3-4^{\circ}$ PU. An operative debridement including necrosectomy was carried out. After treating the wound to achieve clean granulation tissue, plastic surgery was scheduled as soon as the general condition of the patient allowed. We used fasciocutaneous or myofasciocutaneous local flaps, which we performed in VY advancement or rotation technique $[21,22]$. All operations were done by the same team of three surgeons. Postoperatively, the same therapy was applied to every patient. Immobilization was carried out in a special bed (air-fluidized bed) which provides optimal pressure relief. After suture removal and consolidation of the wound, the patient was transferred to an alternating pressure mattress and controlled movement of the hip joint was started and increased gradually. After further stabilization of the soft tissue, an initial 30-minute, therapeutically controlled mobilization to the wheelchair was followed by a careful and controlled increase in sitting time. For all flap surgery patients, the maximum sitting time is limited to four hours in our department until a stable scarring situation is achieved after 6 months [13].

\section{Statistical analysis}

All statistical analyses were performed using SPSS Statistics 19 (IBM, New York) software. First, baseline characteristics of the two groups (SCI patients suffering from a severe PU during the acute phase and matched controls) were compared for differences using non-matched analysis. For continuous variables, either $t$-tests or Mann-Whitney- $U$ tests were used, depending on whether the data was normally distributed or not (assessed by the Shapiro-Wilk test). Categorical variables (e.g., sex, AIS grade, or neurological levels) were compared using the chi-square and Fisher exact test. Because of the matched design (1:3 ratio; matching for baseline AIS grade, neurological level on admission and age, more detailed description see above), we did a stratified analysis using a conditional logistic regression to test for between-group differences on each outcome parameter of the study. The calculated odds ratios are shown in the tables.

\section{Results}

\section{Study population}

At the Trauma Center Murnau, a total of 841 patients with acute traumatic and non-traumatic SCI were treated in the period 2010-2015. Of these we 28 (3\%) patients required flap surgery in the acute SCI phase due to severe PUs. After applying the in- and exclusion criteria (Table 1) complete data sets were available for 15 patients.

Table 2 shows the demographic and clinical characteristics of the PU and the control group on admission. The average age of all patients was 46 years (SD: 17). Patients showed predominantly motor-complete SCI syndrome (AIS 
Table 2 Demographic and clinical characteristics, results are presented in mean $( \pm \mathrm{SD})$ or in total numbers $(\%) p$-values below the $p<0.05$ level were considered statistically significant

\begin{tabular}{llll}
\hline Variable & PU group & Control group & $p$-value \\
\hline$n$ & 15 & 45 & \\
Age [mean \pm SD] & $46.9 \pm 16.0$ & $46.6 \pm 17.6$ & $p<0.669$ \\
Gender [n(\%)] & & & \\
Male & $15(100 \%)$ & $37(82.2 \%)$ & \\
Female & $0(0 \%)$ & $8(17,8 \%)$ & \\
AIS grade $[n(\%)]$ & & & \\
A & $12(80 \%)$ & $36(80 \%)$ & \\
B & $2(13.3 \%)$ & $6(13.3 \%)$ & \\
C & $1(6.7 \%)$ & $3(6.7 \%)$ & \\
Neurological level & & & \\
Cervical & $8(53.3 \%)$ & $23(51.1 \%)$ & \\
Thoracic & $6(40 \%)$ & $20(44.4 \%)$ & \\
Lumbar & $1(6.7 \%)$ & $2(4.5 \%)$ & \\
TMS [mean \pm SD] acute I & $30.7 \pm 20.9$ & $30.4 \pm 21.9$ & $p<0.972$ \\
SCIM [mean \pm SD] acute I & $13.5 \pm 13.3$ & $12.2 \pm 13.6$ & $p<0,656$ \\
\hline
\end{tabular}

AIS American Spinal Injury Association Impairment Scale, TMS total motor score according to the ISNCSCI protocol, SCIM spinal cord independence measure

A and $\mathrm{B} ; n=12 ; 80 \%$ ). The neurological level consisted of cervical or thoracic injuries. The neurological examinations were carried out in the acute I phase.

In 11 out of 15 patients $(73 \%)$ the PU was already present at the time of admission to our center. The average duration until transfer to our specialized SCI department was 24 days. In the remaining four patients, the PU appeared about 32 days after admission to our center. After the diagnosis of PU, the patients were immobilized for about 70 days (range 39-117 d), with positioning in a special airl-fluidized bed (Air-Flow Bed Hillrom) for around 25 days (range $0-101 \mathrm{~d}$ ). On average, it took 83 days (range 45-128 d) until patients could be mobilized into the wheelchair for $2 \mathrm{~h}$ (Table 3 ).

\section{Neurological and functional outcome}

Neurological and functional outcome is provided in Table 4. The PU group showed a significantly lower result in SCIMIII absolute values in the chronic phase (30.9 versus 43.0; $p<0.006)$ and in the increase achieved after 1 year followup (17.4 versus $30.5 ; p<0.006)$ from acute I to chronic. The analysis of the odds ratios for between-group differences (i.e., PU vs. controls) showed that the PU patients not only had a lower OR (0.88; CI 95\% 0.81-0.97) for higher absolute SCIM values during the last assessment, but also a lower OR for a higher difference in SCIM values between first and the last SCIM measurement (0.87; CI $95 \%$ 0.79-0.96).
Table 3 PU specifications according to location grade and size

\begin{tabular}{llll}
\hline Location & Sacrum & Ischium & Major trochanter \\
\hline$[n(\%)]$ & $14(93 \%)$ & $0(0 \%)$ & $1(7 \%)$ \\
EPUAP grade & & $\mathbf{3}$ & $\mathbf{4}$ \\
[n $(\%)]$ & $8(53 \%)$ & $7(47 \%)$ \\
Size & $<\mathbf{~ c m}$ & $\mathbf{5 - 1 0} \mathbf{~ c m}$ & $>\mathbf{1 0} \mathbf{~ c m}$ \\
{$[\mathrm{n}(\%)]$} & $10(67 \%)$ & $3(20 \%)$ & $2(13 \%)$ \\
\hline
\end{tabular}

EPUAP European Pressure Ulcer Advisory Panel

Regarding the neurological recovery (AIS conversion), we examined AIS conversion, that is a change in AIS grade of 1 or 2 steps from acute I to chronic. We did not find a statistically different conversion rate for the PU group (13.3\%) in comparison to the control group $(20.0 \%$; $p<$ $0,603)$. Further, there was no difference in the change of the neurological level $(0.7$ versus $1.0 ; p<0.607)$.

The total motor score (TMS), which expresses the sum of muscle values of the key muscles in the ISNCSCI protocol, showed a trend toward lower values in the PU group (32.8 versus $39.7, p<0.096$ ) and for a lower improvement during the follow-up period ( 2.1 to $9.4 ; p<0.077$ ).

LOS in the PU group was significantly extended by a median of 48 days (range: 147-281d; $p<0.006$ ). PU patients had a slightly higher OR(1.03; CI95\% 1.01-1.06) for a longer LOS than controls.

\section{Discussion}

Many treatment modalities for acute SCI patients are not possible if the patient is immobilized and bedridden. For example mobilization, wheel chair training or automated locomotor training cannot be applied [4, 5]. Moreover training of activities of daily life or intermittent selfcatheterization are postponed [23].

Hence, we investigated the possible influence of severe PU, which necessitates surgical management and prolonged immobilization, in the acute phase after SCI on the neurological and functional outcome.

In our study, patients with severe PUs during acute SCI treatment had a significantly poorer functional outcome as assessed via the SCIM.

The basic principle of PU treatment is pressure relief, which means immobilization and specialized repositioning measures [13]. Thus, PU therapy interferes with key elements in acute SCI therapy, where early mobilization and locomotion training are essential to promote recovery $[4,5]$. These measures must be postponed until the PU is healed. The effective delay of rehabilitation caused by the PU was difficult to identify, since this requires a measurement of the exact healing time of a PU. Even after skin healing intensive 
Table 4 Outcome, results are presented in mean $( \pm \mathrm{SD})$, median (interquartile range), or in total numbers $(\%)$. Bold $p$-values indicate statistical significance at the $p<0.05$ level

\begin{tabular}{|c|c|c|c|c|}
\hline Variable & PU group & Control group & $p$-value & $\begin{array}{l}\text { Odds ratio } \\
\text { (CI 95\%) }\end{array}$ \\
\hline$n$ & 15 & 45 & & \\
\hline AIS grade $[n(\%)]$ & & & $p<0.630$ & \\
\hline A & $10(66.7 \%)$ & $30(66.7 \%)$ & & \\
\hline $\mathrm{B}$ & $3(20.0 \%)$ & $8(17.8 \%)$ & & \\
\hline $\mathrm{C}$ & $2(13.3 \%)$ & $4(8.9 \%)$ & & \\
\hline $\mathrm{D}$ & $0(0 \%)$ & $3(6.7 \%)$ & & \\
\hline AIS conversion $[n(\%)]$ & & & $p<0.630$ & \\
\hline 0 & $13(86.7 \%)$ & $36(80.0 \%)$ & & \\
\hline 1 & $1(6.7 \%)$ & $5(11.1 \%)$ & & \\
\hline 2 & $1(6.7 \%)$ & $4(8.9 \%)$ & & \\
\hline Difference neurological level [mean \pm SD] & $0.7 \pm 1.0$ & $1.0 \pm 1.6$ & $p<0.607$ & \\
\hline $\begin{array}{l}\text { Total motor score }[\text { mean } \pm \mathrm{SD}] \\
\text { chronic phase }\end{array}$ & $32.8 \pm 19.2$ & $39.7 \pm 22.6$ & $p<0.096$ & $0.9(0.85-1.01)$ \\
\hline $\begin{array}{l}\text { Difference motor score }[\text { mean } \pm S D] \text { (acute I } \\
\text { to chronic) }\end{array}$ & $2.1 \pm 3.1$ & $9.4 \pm 15.6$ & $p<0.077$ & $0.83(0.68-1.02)$ \\
\hline SCIM $[$ mean \pm SD) $]$ chronic phase & $30.9 \pm 24.8$ & $43.02 \pm 2.3$ & $p<0.006$ & $0.88(0.81-0.97)$ \\
\hline $\begin{array}{l}\text { Difference SCIM }[\text { mean } \pm S D \text { ] (acute I to } \\
\text { chronic) }\end{array}$ & $17.4 \pm 14.9$ & $30.5 \pm 15.0$ & $p<0.006$ & $0.87(0.79-0.96)$ \\
\hline Length of stay (days) [mean SD] & $194 \pm 38$ & $146 \pm 46$ & $p<0.006$ & $1.03(1.01-1.06)$ \\
\hline
\end{tabular}

AIS American Spinal Injury Association Impairment Scale, SCIM spinal cord independence measure wound healing takes place in the deeper tissue. For this reason, successful "PU healing" was defined for this study by attaining two hours of continuous sitting in the wheelchair, since this requires resilient soft tissue. Thus, in our patients, further treatment and mobilization was delayed for about 83 days until rehabilitative measures could be continued. This also results in a significantly longer total LOS for PU patients. In our cohort, the longer hospital treatment did not compensate for the detrimental effect on functional outcome (Fig. 1). The LOS may be influenced by confounding factors including previous illnesses, concomitant injuries or other medical conditions such as pneumonia, urinary tract infection and other complications [8], which were not assessed in our study.

PU flap surgery is associated with a high rate of complications and recurrence [24, 25]. Thus a comprehensive post-operative treatment protocol is necessary, which heavily interferes with most aspects of functional training for acute SCI patients. According to our PU treatment protocol, patients are immobilized from the diagnosis of $\mathrm{PU}$ until about 4-8 weeks after flap surgery depending on the healing process. There is limited evidence about the length of bedrest after PU surgery but most guidelines and studies propose a 3-6 week period [25]. Following our postoperative protocol it took on average 83 days (range 45-128 d) until PU patients could be mobilized into the wheelchair for $2 \mathrm{~h}$. Continuous sitting for more than $4 \mathrm{~h}$ and hip flection over $100^{\circ}$ is not advised for 6 months post-surgery. Because of these restrictions, mobilization measures are postponed



Fig. 1 Impaired functional recovery for the PU group despite increased LOS. Despite increased LOS by a median of 48 days (range: $147-281 \mathrm{~d} ; p<0.006)$ functional recovery measured by SCIM from acute I to chronic is impaired (difference SCIM acute I to chronic: 17.4 versus $30.5 ; p<0.006$ )

and limited. Bladder management is postponed because patients can only learn to use self-catheterization after proper hip flexion is established; bowel management is postponed because the use of toilet wheelchairs is only allowed after $2 \mathrm{~h}$ of sitting time are established. Training of dressing, grooming and other activities of daily life are equally limited. These points might contribute to the explanation of the functional difference. In this study we 
cannot discriminate between possible negative effects of PUS on neurological outcome and the impact of the strict postoperative protocol. Further studies are needed, to find out if functional training and rehabilitation can be modified to improve functional recovery for PU patients. One possibility would be to implement a less strict post-operative protocol with a sitting program after three weeks to prevent functional loss. The indication for surgery should be assessed with scrutiny, but in stage 3-4 PUs non surgical management has the same need for immobilization as surgical treatment and often leads to instable scarring and recurrence [13].

The follow-up period of this study is only one year. Further studies are necessary to determine if the occurrence of a PU requiring flap surgery also leads to long term consequences such as recurring PUs and other complications. For even after proper healing of the flap, a scar in the pressure exposed soft tissue continues to reduce sitting time of the patient, and this makes them prone to develop further pressure ulcers. Having had one PU is a well-known risk factor for developing further PUs [11, 26, 27].

One other study which has also examined the effects of complications such as PUs during acute SCI treatment on functional outcome and has reported similar results. It showed that in acute SCI patients in Canadian Level 1 trauma centers the PU rate was $30.2 \%$ for tetraplegic patients and $20.0 \%$ for paraplegic patients. In a 6-month follow-up, the patients with tetraplegia, who had complications and longer acute care hospitalization, showed a worse functional outcome in SCIM. The study suggests that complications during the acute phase may influence the functional outcome as far as six months post injury [8].

In our study cohort the prevalence of severe PUs (EPUAP $3-4^{\circ}$ ) with flap surgery during the acute phase of SCI was $3.3 \%$. Far more common were lower grade PUs, especially in the sacral area. These are treated without surgery but also with immobilization and bedrest. In our hospital we try to compromise between the best PU treatment strategy and early mobilization to restart the rehabilitation process and functional training. Special treatment strategies are implemented like botulinum toxin injection to reduce sweating in the affected areas. Thus strict prevention of PUs seems to remain the best strategy. The impact on acute therapy needs to be studied further $[9,28]$.

Nearly all PUs in our study population $(n=14 ; 93 \%)$ were located over the sacrum and were probably caused by lying prone on the back without proper repositioning $[13,26,27]$. Especially longer transport on a spine board or vacuum mattress systems can contribute to sacral PU development $[29,30]$.

Our results underscore that early complications are detrimental for functional outcome after SCI and increase LOS.
Others have shown that an early transfer of patients with traumatic SCI to an integrated multi-disciplinary specialized SCI center decreases the total amount and severity of complications, LOS and overall mortality $[9,27,31]$.

\section{Limitations}

Several limitations need to be acknowledged. First a relatively small number of patients met the inclusion criteria during the 6-year study period. Apart from the severity of the PUs included in this study, another reason for this low patient number is the single center design. This study design provided us with a patient population which received exactly the same care for PUs; however, it would be very interesting to validate our findings in multicenter studies. The retrospective setting allowed us to only include 15 patients with sufficient follow-up data out of 28 affected patients.

Due to the limited number of patients in our database, we did not include gender in the matching process. Otherwise we would not have been able to also match for AIS grade and neurological level.

\section{Outlook}

No single risk factor, apart from injury severity and pneumonia, has been identified for the occurrence of PUs in acute SCI [10, 11, 21, 22]. For this reason, prevention of PUs is necessary at all treatment steps beginning at the accident site. Transport of SCI patients should be done with special care to soft tissue [32]. All hospitals treating SCIs should be aware of the problem and provide a comprehensive PU prevention plan including trained staff, repositioning schedules, specialized mattresses and cushions and visual control of the PU-prone areas [33].

\section{Conclusion}

PUs are a severe complication after acute SCI. Our results show that high grade PUs in the early phase after an acute SCI impair the functional outcome, which cannot be compensated by the longer hospitalization. Even though the present study focuses on the acute phase after SCI, it has to be stressed that prevention of PUs is necessary at all points of care for SCI patients. All involved caregivers should be aware of this problem and provide a comprehensive PU prevention plan. SCI patients should be transferred to specialized centers as soon as possible in order to minimize the risk for SCI-specific complications such as PUs. The results of this study show the importance of developing adjusted protocols for patients with acute SCI and PU. 


\section{Data archiving}

The datasets generated and analyzed during the current study are available from the corresponding author on reasonable request.

Acknowledgements We thank all colleagues who were involved in patient care.

Author contributions DM contributed to the planning of the project, data collection, data analysis, and was the main author of the manuscript. GL contributed to the planning of the project, data collection, data analysis, and was the main reviewer of the manuscript. KB provided data analysis and revision of the manuscript. MV contributed to planning of the project and revision of analysis and the manuscript. MO contributed to planning of the project and revision of analysis and the manuscript. VM contributed to planning of the project and revision of analysis and the manuscript. MD contributed to planning of the project and revision of analysis and the manuscript. SD contributed to planning of the project and data analysis, review of data analysis and revision of the manuscript. All authors read and approved the final manuscript.

\section{Compliance with ethical standards}

Conflict of interest The authors declare that they have no conflict of interest.

Statement of ethics After reviewing the study protocol, the responsible ethical committee of the Bavarian Medical Board approved of the study (2017-040).

Publisher's note: Springer Nature remains neutral with regard to jurisdictional claims in published maps and institutional affiliations.

\section{References}

1. Fehlings MG, Vaccaro A, Wilson JR, Singh A, W Cadotte D, Harrop JS, et al. Early versus delayed decompression for traumatic cervical spinal cord injury: results of the Surgical Timing in Acute Spinal Cord Injury Study (STASCIS). PLoS ONE. 2012;7:e32037.

2. Grassner L, Vogel M, Bühren V, Strowitzki M, Vastmans J, Maier $\mathrm{D}$, et al. Early decompression $(<8 \mathrm{~h})$ after traumatic cervical spinal cord injury improves functional outcome as assessed by spinal cord independence measure after 1 year. J Neurotrauma. 2016;33:1-9.

3. Hadley MN, Walters BC. Introduction to the guidelines for the management of acute cervical spine and spinal cord injuries; guidelines for the management of acute cervical spine and spinal cord injuries. Neurosurgery. 2013;72:3. 1: 4-252

4. Sandrow-Feinber H, Houlé J. Exercise after spinal cord injury as an agent for neuroprotection, regeneration and rehabilitation. Brain Res. 2015;1619:12-21.

5. Wirz M, Mach O, Maier D, Benito-Penalva J, Taylor J, Esclarin A, et al. Effectiveness of automated locomotor training in patients with acute incomplete spinal cord injury: a randomized, controlled, multicenter trial. J Neurotrauma. 2016. https://doi.org/10. 1089/neu.2016.4643

6. Gelis A, Dupeyron A, Legros P, Benaim C, Pelisser J, Fattal C. Pressure ulcer risk factors in persons with SCI: part I: acute and rehabilitation stages. Spinal Cord. 2009;47:99-107.
7. Grossmann R, Frankowski R, Burau K, Toups E, Commett J, Johnson $\mathrm{M}$, et al. Incidence and severity of acute complications after spinal cord injury. J Neurosurg Spine. 2012;17:119-28.

8. Richard-Denis A, Feldman D, Thompson C, Mac-Thiong J. Prediction of functional recovery 6 months following traumatic spinal cord injury during acute care hospitalization. J Spinal Cord Med. 2017. https://doi.org/10.1080/10790268.2017.1279818.

9. Parent S, Barchi S, LeBreton M, Casha S, Fehlings M. The impact of specialized centers of care for spinal cord injury on length of stay, complications, and mortality: a systematic review of the literature. J Neurotrauma. 2011;28:1363-70.

10. Brienza D, Krishnan S, Karg P, Sowa G, Allegretti AL. Predictors of pressure ulcer incidence following traumatic spinal cord injury: a secondary analysis of a prospective longitudinal study. Spinal Cord. 2018;56:28-34.

11. Salzberg CA, Byrne DW, Cartyen CG, Kabir R, van Niewerburgh $\mathrm{P}$, Viehbeck M, et al. Predicting pressure ulcers during initial hospitalization for acute spinal cord injury. Wounds. 1999;11: 45-57.

12. Lala D, Dumont F, Leblond J, Houghton P, Noreau L. Impact if pressure ulcers on individuals living with a spinal cord injury. Arch Phys Med Rehabil. 2014;95-12:2312-9.

13. Rieger U, Scheufler O, Schmid D, Zweifel-Schlatter M, Kalbermatten D, Pierer G. Die sechs Behandlungsprinzipien des Basler Dekubituskonzepts. Handchir Mikrochir Plast Chir. 2007;39: 206-14.

14. Richard-Denis A, Beausejour M, Thompson C, Nguyen B, MacThiong JM. Early predictors of global functional outcome after traumatic spinal cord injury: a systematic review. J Neurotrauma. 2018;35:1705-25.

15. Post M, Dallmeijer A, Angenot E, von Asbeck F, van der Woude L. Duration and functional outcome of spinal cord injury rehabilitation in the Netherlands. J Rehabil Res Dev. 2005;42:75-85.

16. NPUAP / EPUAP / PPPIA. Prevention and treatment of pressure ulcers: quick reference guide. 2014. (second edition).

17. Schuld C, Franz S, Brüggemann K, Heutehaus L, Weidner N, Kirshblum SC, et al. International standards for neurological classification of spinal cord injury: impact of the revised worksheet (revision 02/13) on classification performance. J Spinal Cord Med. 2016;39:504-12.

18. Catz A, Itzkovich M, Agranov E, Ring H, Tamir A. SCIMspinal cord independence measure: a new disability scale for patients with spinal cord lesions. Spinal Cord. 1997;35:850-6.

19. Itzkovich M, Gelernter I, Biering-Sorensen F, Weeks C, Laramee MT, Craven BC, et al. The Spinal Cord Independence Measure (SCIM) version III: reliability and validity in a multi-center international study. Disabil Rehabil. 2006;29:1926-33.

20. Itzkovich M, Shefler H, Front L, Gur-Pollack R, Elkayam K, Bluvshtein V, et al. SCIM III (Spinal Cord Independence Measure version III): reliability of assessment by interview and comparison with assessment by observation. Spinal Cord. 2018;56:46-51.

21. Mortenson WB, Miller WC, The SCIRE Resarch team. A review of scales for assessing the risk of developing a pressure ulcer in individuals with SCI. Spinal Cord. 2008;46:168-75.

22. Verschueren JH, Post MW, de Groot S, van der Woude LH, van Asbeck FW, Rol M. Occurrence and predictors of pressure ulcers during primary in-patient spinal cord injury rehabilitation. Spinal Cord. 2011;49:106-12.

23. Goetz LL, Cardenas DD, Kennelly M, Bonne Lee BS, Linsenmeyer $\mathrm{T}$, Moser $\mathrm{C}$, et al. International spinal cord injury urinary tract infection basic data set. Spinal Cord 2013;51:700-4. Sep

24. Sameem M, Au M, Wood T, Farrokhyar F, Mahoney J. Fasciocutaneous, and perforator-based flaps for treatment of pressure sores. Plast Reconstr Surg. 2012;130:67e-77e. 
25. Tchanque-Fossuo C, William M, Kuzon M. An Evidence-based approach to pressure sores. Plast Reconstr Surg. 2011;127: 931-39.

26. Richardson R, Meyer P Jr. Prevalence and Incidence of pressure sores in acute spinal cord injuries. Paraplegia. 1981;19:235-47.

27. Haisma MD, van der Woude LH, Stam HJ. Complications following spinal cord injury: occurrence and risk factors in a longitudinal study during and after inpatient rehabilitation. J Rehabil Med. 2007;39:393-8.

28. Maharaj MM, Stanford RE, Lee BB, Mobbs RJ, Marial O, Schiller M, et al. The effects of early or direct admission to a specialised spinal injury unit on outcomes after acute traumatic spinal cord injury. Spinal Cord. 2017;55:518-24.

29. James M, Keith LJ, Raymond F, Brett AF. Effect of vacuum spine board immobilization on incidence of pressure ulcers during evacuation of military casualties from theater. Spine J. 2013;13: $1801-8$
30. Pernik M, Seidel H, Blalock R, Burgess A, Horodyski M, Rechtine $\mathrm{G}$, et al. Comparison of tissue-interface pressure in healthy subjects lying on two trauma splinting devices: the vacuum mattress splint and long spine board. Injury. 2016;47:1801-5.

31. Richard-Denis A, Ehrmann Feldman D, Thompson C, BourassaMoreau É, Mac-Thiong JM. Costs and length of stay for the acute care of patients with motor-complete spinal cord injury following cervical trauma: the impact of early transfer to specialized acute SCI center. Am J Phys Med Rehabil. 2017;96:449-56.

32. Mok J, Jackson K, Fang R, Freedman B. Effect of vacuum spine board immobilization on incidence of pressure ulcers during evacuation of military casualties from theater. Spine J. 2013;13: 1801-8.

33. Scovil CY, Flett HM, McMillan LT, Delparte JJ, Leber DJ, Brown $\mathrm{J}$, et al. The application of implementation science for pressure ulcer prevention best practices in an inpatient spinal cord injury rehabilitation program. J Spinal Cord Med. 2014;37:589-97. 